\title{
INTRANASAL TREATMENT IN LEPROSY
}

\section{E. MU1R}

Among the most distressing complications of leprosy, and especially of the lepromatous type, are certain conditions of the nasal cavities. These vary from irritating dryness and epistasis to discharge of crusts and pus, and partial or even total obstruction of air passage. The paranasal sinuses may be infected, adding to the distress of the patient and increasing septic absorption. These conditions are due partly to the lepromatous infiltration, but still more to secondary infection.

In planning treatment three objects must be kept in mind: the prevention of sepsis, the removal of sepsis if it has occurred, and the clearing up of leproma.

I. The prevention of sepsis. Septic absorption causes deterioration of the patient's health and exacerbates the leprous condition. It is important therefore to take steps from the outset of leprosy to safeguard nasal hygiene. In doing this it is well to avoid strong antiseptics or drugs which would lower the resistance of the mucous membrane. This is the more important as, whatever treatment is adopted, it may have to be continued regularly for years. Also for this reason it is necessary that the treatment be a simple one which the patient himself can carry out daily. The following simple method is adapted from that recommended by Parkinson*.

* Non-traumatic Ventilation Treatment of the Nose and Sinuses, S. N. Parkinson, Jl. Larj'ng. and Otol. Oct., 1939. 
The simple requirements are a small pipette and a bottle of normal saline which the patient can prepare himself by boiling a teaspoonful of salt in a pint of water, and bottling the same. If there is much nasal catarrh or blocking of the entrances to the sinuses, then 0.9 per cent ephedrine hydrochloride can be added.

The patient lies sideways on a couch (or the floor) with a pillow under the lower arm, and the shoulder projecting beyond the pillow. The head is bent towards the lower shoulder so that the vertex rests on the couch. The patient keeps his mouth open and breathes audibly so as to avoid breathing through the nose or swallowing. About a drachm of the solution is pipetted into each nostril and is held there for two minutes. The nares are then occluded with the fingers, the mouth is shut, and strong inspiratory movements are made two or three times so as to form each time powerful negative pressure in the nose. The effect of this is to suck the air out of the sinuses, and, as the entrances to these are submerged, the saline rushes into the sinus each time the suction is relaxed. The saline finds its way out gradually by ciliary vection and thereby washes out and drains these cavities. Ephedrine, if added, removes congestion from the mucous membrane and makes patent the entrances to the sinuses if these are blocked, so that the solution can enter more readily.

Normal saline causes no irritation, and with this form of lavage carried out two or three times a day, septic complications, so apt to occur in leprosy, can be avoided.

2. If severe sepsis has occurred and the nose is blocked with pus and crusts, another modification of the same method may be tried. The patient lies in the same position and uses the same solution with or without ephredine, only it is necessary to retain the saline in the nose for a much longer period and to renew it several times so as to soften the crusts thoroughly; then they can be removed with a saline douche. Until sepsis is cleared up the second part of the process-sucking the solution into the sinuses-should be omitted. In very septic cases sodium sulphate solution may be substituted for normal saline, the solution being Io per cent or as strong as the patient can bear without irritation. The patient may spend a considerable part of the day, according to tolerance, in carrying out this treatment until sepsis has been removed.

3. The above methods aim at preventing or removing sepsis. For dealing locally with leproma, cauterization in one form or another is best. One of the simplest, requiring no special apparatus, is the application of trichloracetic acid solution. Before 
cauterising the nose (a) remove sepsis if present, and (b) improve the general health. The most suitable patients are $\mathrm{L}_{2}$ or $\mathrm{L}_{3}$ cases with strongly positive nasal smears, who are in good general health and in whom leprosy throughout the body is yielding favourably to treatment or at least not advancing.

The mucous membrane is anaesthetised with $2 \%$ novocaine solution, applied as spray and by stuffing cotton wool soaked in the anaesthetic up the nostrils. A ten per cent solution of the acid is carefully applied with a fine brush to the mucous membrane, a speculum and a good light being used to make sure that all the lining is bleached as high as possible. The application may be repeated after two weeks. After anaesthetising, and before each application of acid, remove a small piece of mucous membrane with a sharp pointed instrument and make a smear for examination. Progress can thus be noted.

Careful and timely treatment of the nose should avoid deformity which results from destruction of the septum and contraction of the scars of septic ulcers. Attention to the nose also diminishes to a large extent the danger of the discharge of $M$. leprae and the spread of infection. 